\title{
Final Report for Colorado State University
}

\author{
Representation-Independent Compiler Technology for Domain-Specific \\ Analysis with the OpenAnalysis Toolkit (DE-FG02-06ER25724) \\ PI at Colorado State University: Michelle Mills Strout \\ PI at Argonne National Laboratory: Paul D. Hovland \\ Program Manager: Fred Johnson
}

Compiler infrastructures are monolithic in design, and one cannot readily use any of the components without adopting the entire infrastructure. One crucial component is program analysis. However, since each compiler infrastructure's analyses are heavily integrated with its own unique intermediate representation (IR), the analyses cannot easily be extracted, and much duplicate effort occurs for each new infrastructure. Through the development of the OpenAnalysis toolkit, we are exploring a novel design strategy for program analysis software that introduces a new level of abstraction between analysis algorithms and the intermediate representations that they manipulate. Another important objective is to enable domain experts to easily specify domain-specific program analyses, thus reducing the program analysis learning curve for a broad spectrum of researchers, including computational scientists that need domain-specific analysis for program transformations such as parallelization, numerical optimization, and automatic differentiation.

At Colorado State University, the OpenAnalysis project was supported through DOE grant DE-FG02-06ER25724 from March 2006 through February 2010. The OpenAnalysis project has contributed in the following main results:

1. a public domain suite of software for program analysis (i.e. OpenAnalysis, UseOA-ROSE, UseOA-Open64) that is currently being used in software tools being developed at Argonne,

2. the development of linearity analysis for use in improving the performance of the adjoint code generated by automatic differentiation tools,

3. a domain-specific programming language based on set building notation for specifying dataflow analyses at a high-level of abstraction with a compiler that generates the full data-flow analysis implementation,

4. theory and a prototype implementation for performing data-flow program analysis of MPI programs,

5. and the development of a parallel, network protocol simulation framework (which turns out to be similar to data-flow analysis frameworks) in collaboration with networking researchers at CSU.

The above research contributions have resulted in 10 conference and workshop publications $[17,16$, $4,14,6,8,9,12,13,5], 2$ journal papers [19, 11], a master's thesis [10], a conference paper still in submission [2], and a journal paper under preparation [15]. At Colorado State University, this project supported one graduate student for four years, provided some summer salary for the PI, and provided some hourly support for an undergraduate research assistant.

The remainder of this report provides more details about the main results from the OpenAnalysis project and also summarizes some of the lessons learned. 


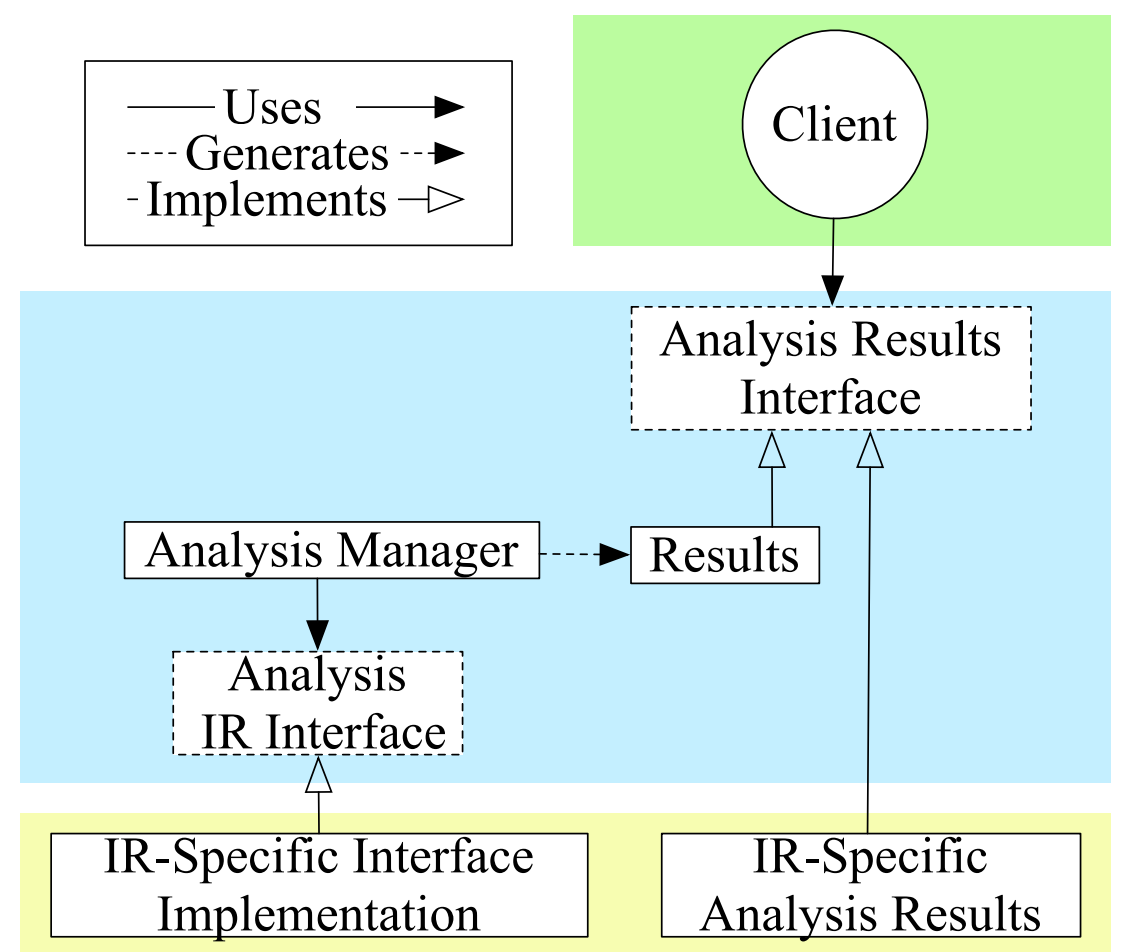

Figure 1: Software Architecture for OpenAnalysis

\section{The OpenAnalysis Toolkit}

The OpenAnalysis Toolkit is a software package capable of performing program analyses such as alias/pointer analysis, side-effect analysis, control-flow graph construction, call graph construction, interprocedural control-flow graph (ICFG) construction, the construction of interprocedural controlflow graphs with communication edges so as to model MPI programs (MPI-ICFG), and data-flow analyses such as reaching constants, reaching definitions, liveness, and activity analysis. OpenAnalysis performs the analyses by making queries to analysis-specific interfaces. These interfaces are then implemented for each intermediate representation that we would like to analyze. Figure 1 shows the software architecture for OpenAnalysis.

OpenAnalysis is currently connected to the Open64 compiler being developed at RICE [7] and the ROSE compiler infrastructure [1] being developed at LLNL. OpenAnalysis is also being used in the HPCtoolkit [3] being developed at RICE. We have also incorporated OpenAnalysis into the Simple Compiler (SC), which is a compiler for an tiny subset of the C language. We are using the connection with $\mathrm{SC}$ as a basis for the OpenAnalysis user documentation.

OpenAnalysis, the interface between OpenAnalysis and ROSE, the interface between OpenAnalysis and Open64, and SC can all be downloaded from the OpenAnalysis web page [18].

\section{Linearity Analysis}

Using OpenAnalysis as an implementation framework, we developed various domain-specific program analyses for use within automatic differentiation tools. Linearity analysis is one such analysis that is useful in automatic differentiation and in numerical optimization algorithms. Linearity anal- 


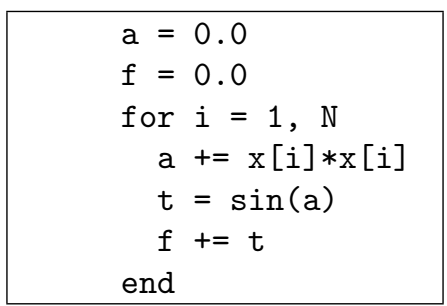

Figure 2: Example loop

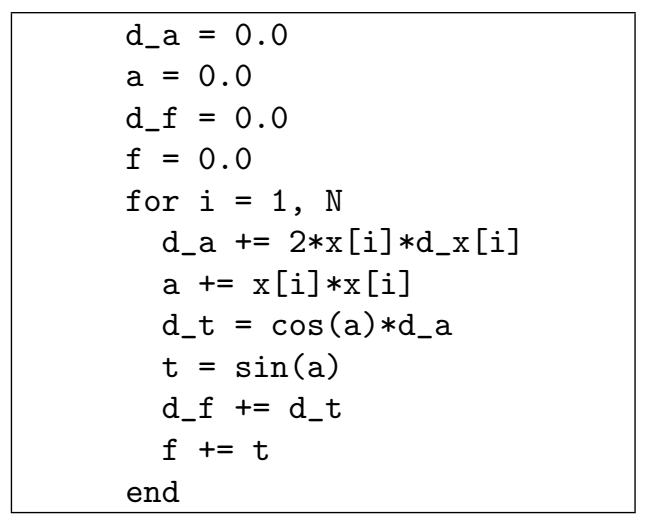

Figure 3: Example loop after forward mode $\mathrm{AD}$

ysis determines whether the dependence between two variables is nonexistent, linear, or nonlinear. A variable is said to be linearly dependent on another if all of the dependences along all of the dependence chains are induced by linear or affine functions (addition, subtraction, or multiplication by a constant). A variable is nonlinearly dependent on another if a nonlinear operator (multiplication, division, transcendental functions, etc.) induces any of the dependences along any of the dependence chains.

One application of linearity analysis is the optimization of derivative code generated by automatic differentiation $(\mathrm{AD})$ via the reverse mode. $\mathrm{AD}$ is a technique for transforming a subprogram that computes some function into one that computes the function and its derivatives. AD works by combining rules for differentiating the intrinsic functions and elementary operators of a given programming language with the chain rule of differential calculus. One strategy, referred to as the forward mode, is to compute partials as the intrinsic functions are evaluated and to combine the partials as they are computed. For example, forward mode AD transforms the loop in Figure 2 into the code in Figure 3. Reverse mode AD results in less computation in the derivative code if the number of independent variables is much larger than the number of dependent variables. Figures 4 shows the adjoint code after applying the reverse mode. Notice that the temporary variable a must be promoted to an array to store results needed in the adjoint computation.

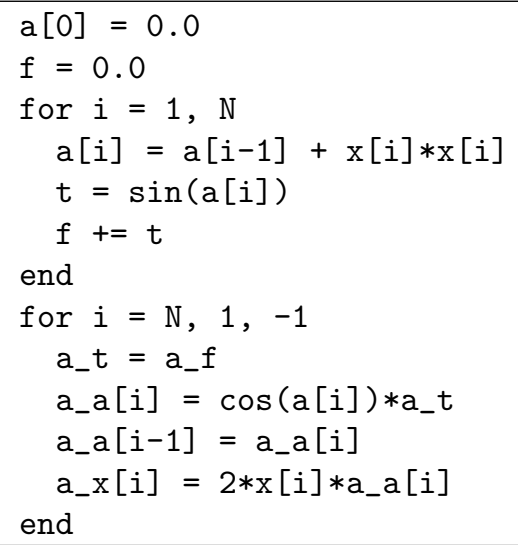

Figure 4: Example loop after reverse mode automatic differentiation

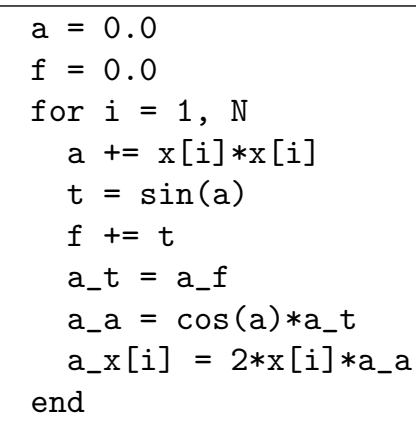

Figure 5: Adjoint code after reversing the adjoint loop and fusing it with the original computation

One of the many applications of this analysis is determining whether a loop involves only linear loop-carried dependences and therefore the adjoint of the loop may be reversed and fused with the computation of the original function thus saving space and computation. In [14], we specify 

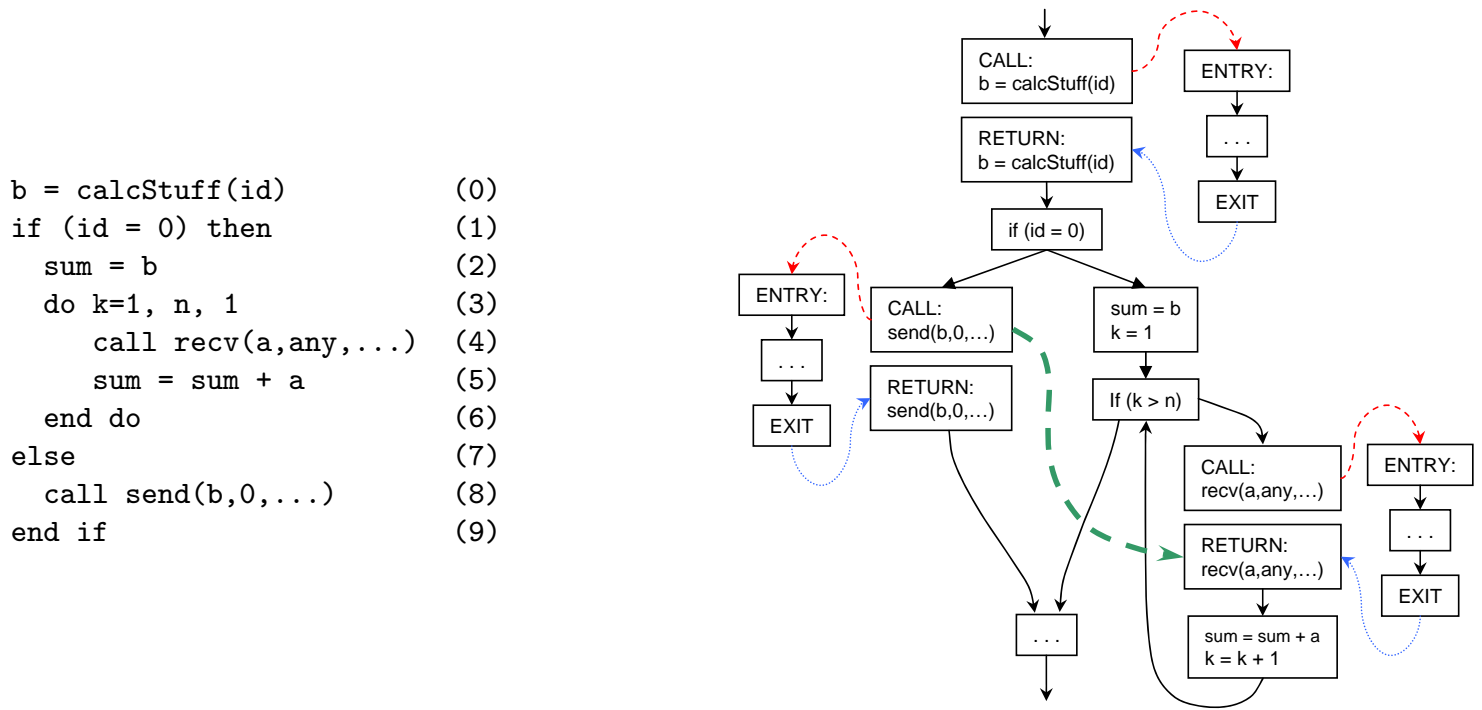

Figure 6: Example MPI-ICFG.

the data-flow equations that compute linearity analysis. In addition, we describe using linearity analysis with array dependence analysis to determine whether a loop-carried dependence is linear or nonlinear.

\section{The Data-Flow Analysis Generator}

Linearity analysis [14] as well as other analyses we wrote by hand in OpenAnalysis such as activity analysis $[8,9]$ are examples of data-flow analyses. Data-flow analysis is a common technique for gathering program information for use in program transformations such as register allocation, deadcode elimination, common subexpression elimination, and scheduling. Current tools for generating data-flow analysis implementations enable analysis details to be specified orthogonally to the iterative analysis algorithm but still require implementation details regarding the may and must use and definition sets that occur due to the effects of pointers, side effects, arrays, and user-defined structures.

As part of the OpenAnalysis project, we developed a Data-Flow Analysis Generator tool (DFAGen), which enables analysis writers to generate analyses for separable and nonseparable data-flow analyses that are pointer, aggregate, and side-effect cognizant from a specification that assumes only scalars $[12,13]$. By hiding the compiler-specific details behind predefined set definitions, the analysis specifications for the DFAGen tool are typically less than ten lines long and similar to those in standard compiler textbooks. The main theoretical contribution of this work is the automatic determination of when to use the may or must variant of a predefined set usage in the analysis specification.

\section{Data-Flow Analysis for MPI Programs}

In addition to analyzing serial programs, we also developed techniques for analyzing parallel programs written using MPI. Message passing via MPI is widely used in parallel programs executing under the SPMD model. To perform nonseparable data-flow analyses for such programs, it is necessary to model the semantics of message-passing. In $[16,5,15]$, we developed a method for 
performing data-flow analysis on the MPI-ICFG representation that leverages existing data-flow analysis techniques with a separate propagation over communication edges, which model the flow of data between message sends and receives. Figure 6 contains an example MPI-ICFG with control flow edges represented as arrows with solid lines, call and return edges represented as dotted lines, and a communication edge represented with a dashed line.

Our results showed that data-flow analysis performed in this fashion converges in a reasonable number of steps and improves the accuracy of reaching constants and a domain-specific analysis called activity analysis. Additionally, we developed a context-sensitive alias analysis, approaches for reducing the number of communications edges in the MPI-ICFG, and algorithms for determining the depth of the MPI-ICFG.

\section{Applying Data-Flow Analysis Concepts to Network Pro- tocol Simulations}

Our experiences with data-flow analyses led to an interesting tangent where we teamed up with some networking researchers in the computer science department at Colorado State University to apply data-flow analysis techniques to the routing protocol simulation problem. Modern routing protocols for the internet implement complex policies that take more into account than just path length. However, current routing protocol simulators are limited to either working with hard-coded policies or working on small networks (1000 nodes or less). It is currently not possible to ask questions about how the routing tables will change on all of the autonomous systems (e.g., AT\&T, Sprint, etc.) in the internet, given a change in the routing protocol.

We developed a routing policy simulation framework called MR.Sim that enables such simulations to be done on resources that are readily available to researchers, such as a small set of typical desktops [13, 2]. We base the policy simulation framework on the Routing Algebra Meta-Language (RAML), which is a formal framework for specifying routing policies. Our simulator is able to scale to large scale network graphs (10K - 100K nodes) while taking on the order of 45 minutes to perform a full simulation. Our theoretical contributions include proving that the signatures and the meet operation induced by the preference operator in RAML define a semilattice and that routing policy simulation frameworks are analogous to data-flow analysis frameworks.

There are many ways our work can be expanded. Future work includes: (1) looking at methods to aid in load balancing and therefore achieve further performance in multicore machines and clusters, (2) expanding the policy implementation framework to include more of the features of RAML (such as new ways of composing algebras), (3) using the tool to study various networking questions related to the affects of topology and policy, and (4) further studying the connection between data-flow analysis and metarouting. Many performance optimizations for data-flow analysis frameworks have been developed over the years: interval analysis, bit-vectors, etc. Although some of these approaches require properties that label operators and network topologies do not have (i.e., interval analysis is a no-go due to non-reducibility in the graph and lack of DFA monotonicity), other performance optimizations could be developed to further improve the performance of the routing simulation.

\section{Conclusions}

The OpenAnalysis project funded with DOE grant DE-FG02-06ER25724 resulted in a number of important software and research results including the OpenAnalysis toolkit, which will continue being used in automatic differentiation tools such as ADIC and OpenAD/FortTK [19]. The approach of querying Intermediate Representations (IRs) in an analysis-specific way ran into difficulties in that the higher-level IRs such as the ROSE abstract syntax tree did not explicitly expose language se- 
mantics. As such, the IRInterfaces essentially had to convert the AST into a lower-level intermediate representation for analysis purposes while still maintaining the connections with the high-level AST. Therefore, as a general approach analysis-specific queries to a high-level AST will probably require more requirements on the AST representation so as to enable rigorous testing and less complex IR interface implementations.

In addition to the OpenAnalysis toolkit, this research has resulted in theoretical contributions for automating the generation of data-flow analysis implementations, analyzing adjoint programs in the context of automatic differentiation, and analyzing MPI programs. Our experience with data-flow analysis automation led to breakthroughs in network protocol simulation frameworks as well.

\section{References}

[1] Dan Quinlan. Rose compiler. http://www.rosecompiler.org/.

[2] S. DiBenedetto, A. I. Stone, M. M. Strout, and D. Massey. Simulating internet scale topologies with metarouting. Technical report, Technical Report CS-10-103 Colorado State University, March 2010.

[3] John Mellor-Crummey. Hpctoolkit. http://hpctoolkit.org/.

[4] B. Kreaseck, L. Ramos, S. Easterday, M. M. Strout, and P. Hovland. Hybrid static/dynamic activity analysis. In Proceedings of the 3rd International Workshop on Automatic Differentiation Tools and Applications (ADTA'06), Reading, Englandications (ADTA'06), Reading, England, May 2006.

[5] B. Kreaseck, M. M. Strout, and P. Hovland. Depth analysis of mpi programs. In Proceedings of The First Workshop on Advances in Message Passing (co-located with PLDI 2010 ), June 6 2010.

[6] U. Naumann, J. Utke, , C. Wunsch, C. Hill, P. Heimbach, M. Fagan, N. Tallent, and M. Strout. Adjoint code by source transformation with OpenAD/F. In Proceedings of the European Conference on Computational Fluid Dynamics (ECCOMAS CFD 2006). TU Delft, 2006.

[7] Rice University. Open64 project. http://www.hipersoft.rice.edu/open64/.

[8] J. Shin and P. D. Hovland. Comparison of two activity analyses for automatic differentiation: Context-sensitive flow-insensitive vs. context-insensitive flow-sensitive. In The 22nd Annual ACM Symposium on Applied Computing (SAC), 2007.

[9] J. Shin, P. Malusare, and P. Hovland. Design and implementation of a context-sensitive, flowsensitive activity analysis algorithm for automatic differentiation. In C. B. et al., editor, Proceeding of The 5th Int'l Conference on AD, Lecture Notes in Computational Science and Engineering 64, pages 115-126, 2008.

[10] A. Stone. Automatic determination of may/must set usage in data-flow analysis. Master's thesis, Colorado State University, Summer 2009.

[11] A. Stone, M. Strout, and S. Behere. May/must analysis and the dfagen data-flow analysis generator. Information and Software Technology, 51(10):1440-1453, October 2009.

[12] A. Stone, M. M. Strout, and S. Behere. Automatic determination of may/must set usage in data-flow analysis. In Proceedings of the Eighth IEEE International Working Conference on Source Code Analysis and Manipulation (SCAM), 2008.

[13] A. I. Stone, S. DiBenedetto, M. M. Strout, and D. Massey. Scalable simulation of complex network routing policies. In Proceedings of the ACM International Conference on Computing Frontiers (CF), May 17-19, 2010. 
[14] M. M. Strout and P. D. Hovland. Linearity analysis for automatic differentiation. In Proceedings of the 3rd International Workshop on Automatic Differentiation Tools and Applications (ADTA'06), Reading, England, May 2006.

[15] M. M. Strout, B. Kreaseck, and P. D. Hovland. The theory and practice of data-flow analysis for mpi programs. In preparation.

[16] M. M. Strout, B. Kreaseck, and P. D. Hovland. Data-flow analysis for MPI programs. In Proceedings of the International Conference on Parallel Processing (ICPP), August 2006.

[17] M. M. Strout, J. Mellor-Crummey, and P. Hovland. Representation-independent program analysis. In Proceedings of the The sixth ACM SIGPLAN-SIGSOFT Workshop on Program Analysis for Software Tools and Engineering (PASTE), September 5-6 2005.

[18] M. M. Strout and A. Stone. The openanalysis toolkit. http://openanalysis.berlios.de/, May 2010.

[19] J. Utke, U. Naumann, M. Fagan, N. Tallent, M. Strout, P. Heimbach, C. Hill, and C. Wunsch. Openad/f: A modular, open-source tool for automatic differentiation of Fortran codes. ACM Transactions on Mathematical Software, 34(4), 2008. 